\title{
Importance socioéconomique des parcs agroforestiers à Elaeis guineensis Jacq. dans la région de Cacheu (Guinée-Bissau)
}

\author{
Boubacar SAGNA ${ }^{1,3^{*}}$, Daouda NGOM ${ }^{2}$, Mamadou Abdoul Ader DIEDHIOU ${ }^{1}$, \\ Boubacar CAMARA ${ }^{1}$, Mamadou GOUDIABY ${ }^{4}$, Abdou Seydou MANE ${ }^{3}$ et \\ Yvan LE COQ
}

\footnotetext{
${ }^{1}$ Laboratoire d'Agroforesterie et d'Écologie, Université Assane SECK de Ziguinchor, BP : 523, Diabir.

${ }^{2}$ Laboratoire d'Écologie végétale, Université Cheikh Anta Diop de Dakar, BP : 5005, Dakar Fann.

${ }^{3}$ Groupe de recherche et de réalisations pour le développement rural (Grdr) : CP 573, Bissau.

${ }^{4}$ Inspection régionale des Eaux et Forêts, Chasse et Conservation des Sols de Ziguinchor.

*Auteur correspondant; E-mail : boubacarsagna22@gmail.com
}

\section{RESUME}

La Guinée-Bissau recèle d'importants parcs agroforestiers à Elaeis guineensis. Ces palmeraies sont très exploitées pour les nombreux biens, services et produits qu'elles procurent aux communautés locales de la région de Cacheu. Cependant l'importance de ces parcs est très peu étudiée et est souvent limitée à quelques services et produits fournis par le palmier à huile. C'est ainsi que la présente étude se fixe pour objectif de contribuer à l'évaluation de l'importance socioéconomique de ces parcs à Elaeis guineensis dans la région de Cacheu. Pour ce faire, un inventaire forestier a été réalisé dans 90 placettes carrées de $2500 \mathrm{~m}^{2}$. En plus, une enquête socioéconomique été réalisée auprès de $15 \%$ de la population active de 14 villages dans lesquels une enquête ethnobotanique a également été faite à travers un focus groupe. Ces différentes méthodes combinées ont permis de constater une richesse spécifique de 75 espèces ligneuses dans les parcs. L'espèce caractéristique des parcs, Elaeis guineensis est exploitée sur toutes ses composantes pour divers usages contribuant ainsi à l'amélioration du bien-être social de la population notamment dans l'alimentation, la santé et l'économie locale. Les autres espèces accompagnatrices ont aussi une importance capitale dans la vie de la population locale. D'autres activités telles que l'agriculture pluviale, l'élevage, la chasse, la production maraîchère, la pharmacopée, les activités culturelles et cultuelles, etc. sont aussi pratiquées dans la palmeraie. D'où la nécessité d'exploiter durablement ces parcs afin de pérenniser tous ces avantages.

(C) 2019 International Formulae Group. All rights reserved.

Mots clés : Services écosystémiques, palmier à huile, exploitation, ressources forestières.

\section{Socioeconomic importance of agroforestry parklands of Elaeis guineensis Jacq. in the region of Cacheu (Guinea-Bissau)}

\author{
ABSTRACT \\ Guinea-Bissau has important agroforestry parklands in Elaeis guineensis. These palm groves are highly \\ exploited for the many goods, services and products they provide to local communities in the Cacheu region. \\ However the importance of these parklands is very little studied and is often limited to some services and
}


products provided by the oil palm. Thus, the objective of this study is to contribute to the evaluation of the socio-economic importance of these Elaeis guineensis parklands in the Cacheu region. To do this, a forest inventory was carried out in 90 square plots of $2500 \mathrm{~m} 2$. In addition, a socio-economic survey was conducted among $15 \%$ of the working population in 14 villages in which an ethnobotanical survey was also conducted through a focus group. These different methods combined revealed a specific richness of 75 ligneous species in the parks. The characteristic species of the parklands, Elaeis guineensis is exploited on all its components for various uses thus contributing to the improvement of the social well-being of the population in particular in the food, the health and the local economy. The other companion species are also of paramount importance in the life of the local population. Other activities such as rainfed agriculture, livestock breeding, hunting, market gardening, pharmacopoeia, religious and cultural activities, etc. are also practiced in the palm grove. Hence the need to sustainably exploit these parks to sustain all these benefits.

(C) 2019 International Formulae Group. All rights reserved.

Keywords: ecosystem services, palm oil, exploitation, forest resources.

\section{INTRODUCTION}

La Guinée-Bissau recèle une importante potentialité en ressources naturelles, particulièrement forestières (Arvanitis, 2014). Ces dernières occupent une place importante dans les moyens de subsistance de la population locale. Parmi les espèces forestières de la zone, Elaeis guineensis (palmier à huile) fait partie des plus exploitées pour ses nombreux services et produits tels que l'huile de palme, l'huile de palmiste, le vin, les matériaux de construction, les médicaments, le savon, les engrais, etc. (Carrere, 2010). C'est pourquoi les populations l'ont maintenu, au cours des siècles, dans leur système traditionnel d'utilisation des terres, celui des « parcs agroforestiers». Un parc agroforestier est « un système d'utilisation des terres dans lequel les végétaux ligneux pérennes sont délibérément conservés en association avec les cultures et/ou l'élevage dans un arrangement spatial dispersé et où existent à la fois des interactions écologiques et économiques entre les ligneux et les autres composantes » (Sagna, 2016). Dans la région de Cacheu située au Nord-ouest de la Guinée Bissau, on rencontre d'importantes formations naturelles de palmiers sous forme de parcs agroforestiers (Banjai, 2009). La production de l'huile de palme et l'exploitation du vin de palme y sont des activités génératrices de revenus pour les familles rurales, particulièrement pour les jeunes et femmes producteurs. Cependant ces parcs sont très peu étudiés et leur importance est souvent réduite à quelques services et produits fournis par le palmier à huile qui est l'espèce caractéristique. Ainsi, la présente étude se fixe pour objectif global de contribuer à l'évaluation de l'importance socio-économique des parcs agroforestiers à Elaeis guineensis dans la région de Cacheu. Il s'agit spécifiquement d'évaluer l'importance socioéconomique du palmier et de déterminer les services écosystémiques fournis par les autres espèces composant la palmeraie.

\section{MATERIELS ET METHODES Enquête}

Pour mieux comprendre la contribution de la palmeraie au bien-être de la population locale, deux types d'enquêtes ont été réalisées : une enquête socioéconomique et une enquête ethnobotanique.

\section{Enquête socioéconomique}

Afin d'identifier l'ensemble des produits et services tirés du palmier à huile, des enquêtes socioéconomiques ont été réalisées dans la zone d'étude. Pour cela, un questionnaire individuel a été administré à un échantillon de $15 \%$ de la population active de chacun des 14 villages composant la zone d'étude. L'âge minimale des enquêtés était fixée à 18 ans. Le choix des enquêtés a été fait en fonction les différents secteurs d'activités dans la palmeraie. Ainsi, les exploitants, les productrices d'huile de palme, les autorités traditionnelles, les consommateurs et les commerçantes d'huile de palme ont été 
interrogés séparément dans chaque village. Au total, 344 personnes ont été interrogées.

\section{Enquête ethnobotanique}

Les relevés de végétation réalisés dans les parcs à Elaeis guineensis en région de Cacheu ont permis d'inventorier les différentes espèces ligneuses composant ces écosystèmes. C'est sur la basse de la liste des espèces recensées qu'une enquête ethnobotanique a été réalisée à travers des focus groupes mixtes organisés dans chacun des six villages retenus. Ces enquêtes ont permis de connaitre les services écosystémiques fournis par chacune des espèces trouvées dans la zone à savoir son niveau d'utilisation par la population locale dans l'alimentation humaine, le bois de chauffe, le bois de service, le bois d'œuvre, le fourrage, la fertilisation, la pharmacopée, etc. Dans la fiche d'enquête élaborée, une échelle allant de 0 à 3 était utilisée pour évaluer le niveau d'utilisation des espèces pour chaque catégorie d'usage : $0=$ pas utilisé, $1=$ peu exploitée, $2=$ moyennement exploitée et $3=$ beaucoup exploitée.

\section{Restitution et validation des résultats}

Après le traitement des données, les premiers résultats ont été présentés lors d'un atelier multi- acteurs de restitution et validation des informations recueillies regroupant les ressortissants des différents sites, les services techniques étatiques (service régional des Eaux et Forêts, service des parcs) et d'autres acteurs de la filière huile de palme en région de Cacheu.

\section{Traitement des données}

Les données d'enquête ont été d'abord dépouillées manuellement puis saisies et traitées à l'aide du logiciel Sphinx Plus. Celuici a permis de générer directement les résultats en fonction des variables de saisie en utilisant les techniques d'analyses uni-variées ou bi-variées. Les premiers résultats ont été transformés sur le tableur Excel pour être présentés sous forme de tableaux, de diagramme et d'histogrammes. Lors du traitement de données d'enquête, les variables suivantes ont été calculées.
$>$ Fréquence de Citations (FC)

Elle est calculée à travers la formule suivante

$$
\mathrm{FC}=\frac{\text { Nombre de citations d'une espéce }}{\text { Nombre total de répondants }} X 100
$$

\section{$>$ Valeur d'usage (VU)}

Pour chaque espèce citée, une valeur d'usage (Use Value ou UV) défini par Phillips et al. (1994) sera définie. La valeur d'usage est une manière d'exprimer l'importance relative de chaque espèce pour la population dans les usages des parcs agroforestiers.

$$
\begin{aligned}
\mathrm{VU}=\frac{\sum \mathrm{U}}{\mathrm{n}} \quad \begin{array}{l}
\mathrm{U}=\text { nombre de citations par } \\
\text { espèce } \\
\mathrm{n}=\text { nombres d'informateurs par } \\
\text { rapport aux usages de l'espèce }
\end{array}
\end{aligned}
$$

\section{Analyse statistique}

Des logiciels comme R. version 3.4.2 (2017-09-28) et XLSTAT ont été utilisés pour calculer certains paramètres ainsi que réaliser des analyses multi-variées. Il s'agit notamment l'Analyse en Composante Principales réalisée en vue de faire une représentation des espèces identifiées dans les parcs en fonction de leur catégorie d'usage.

\section{RESULTATS}

L'analyse de l'importance socioéconomique des parcs à Elaeis guineensis a été réalisée à deux niveaux. Il s'agit de l'identification des produits et services fournis par le palmier à huile et de l'ensemble des usages des autres espèces ligneuses composant la palmeraie.

\section{Mode d'exploitation du palmier à huile dans la région de Cacheu}

Il ressort des enquêtes réalisées auprès des populations locales que l'ensemble des parties du palmier à huile est exploité pour divers usages. Ces différents usages sont consignés dans le Tableau 1.

\section{Les feuilles}

Les utilisations faites à partir des feuilles sont très nombreuses et diverses. Les nervures principales ou les feuilles entières sont utilisées par les populations locales pour la clôture des toilettes traditionnelles, des 
périmètres maraîchers, etc. Dans un contexte local où l'Etat manque de moyens financiers, le palmier à huile joue un rôle important dans l'éducation car les feuilles sont beaucoup utilisées dans la confection d'abris provisoires. Les nervures principales servent également à la fabrication des ceintures utilisées pour grimper les palmiers ou les rôniers. Elles sont également utilisées pour le plafonnage des maisons, pour la fabrication de palissades, lits mais aussi peuvent servir de cordes pour attacher les clôtures ou les fagots de bois. Elles servent aussi à fabriquer de gros paniers utilisés dans la saliculture pour le stockage du sel. La nervure des folioles est utilisée pour la confection des balais. Ceux-ci sont souvent vendus et procurent aux usagers de la palmeraie des revenus monétaires non négligeables. Les folioles sont utilisées par les récolteurs de vin pour confectionner des sortes d'entonnoir servant de conduite au vin du palmier à la bouteille (Figure 1).

\section{Les fleurs}

Les fleurs de palmiers sont utilisées
comme fertilisant dans l'agriculture, principalement en arboriculture. En effet, elles augmentent non seulement la fertilité du sol, mais aussi permet de garder l'humidité pendant plusieurs heures autour des plants. Elles sont aussi utilisées comme insectifuges. En effet, la fumée de la fleur sèche allumée est utilisée pour chasser des insectes comme les abeilles pendant la récolte du miel des ruches traditionnelles, mais aussi pour embaumer les animaux domestiques afin de chasser les moustiques et les mouches. La cendre provenant des fleurs, mélangée avec d'autres produits comme la soude, sert à fabriquer du savon traditionnel.

En plus de son usage dans l'agriculture, dans l'apiculture et l'élevage, certains exploitants commencent d'abord à récolter le vin de palme à partir des fleurs en attendant la maturité des régimes pour continuer sur le même pied. Cela rend très longue la durée de récolte et contribue à la surexploitation du palmier et à l'altération de la qualité des noix et de l'huile de palme

\section{Les régimes}

Les produits provenant du régime comme la pulpe sucrée, l'huile de palme, la sauce palmiste «thében», l'huile de palmiste etc. sont utilisés dans l'alimentation des populations locales (Figure 2).

Pendant la période de maturité des régimes (Février-juin) la fréquence moyenne de consommation de l'huile de palme et de la sauce de palmiste en milieu rural est respectivement estimée à 12 et 9 jours/mois, soit environ tous les trois jours du mois. En saison des pluies, marquée par une abondance de gombo, la consommation d'huile de palme augmente considérablement. Celle-ci contribue à enrichir l'alimentation de la population grâce à sa teneur en vitamine $\mathrm{A}$ (Bêta-carotènes) et E. Cependant, il a été noté une baisse de la fréquence de consommation d'huile de palme chez les consommateurs principalement causées par la cherté de l'huile de palme. En plus de son importance dans l'alimentation, l'huile de palme a une valeur économique très importante. En effet, la vente de cette huile fournit aux exploitants, aux productrices et aux commerçants un revenu annuel pouvant dépasser facilement 750000 f CFA. Ces revenus sont souvent utilisés dans la prise en charge des besoins de la famille notamment en termes de nourriture, d'habillement, de scolarisation des enfants, de santé et d'organisation de cérémonies, etc.

La production de l'huile de palmiste n'est pas très développée dans la région de Cacheu car les populations, majoritairement constituées de chrétiens et d'animistes, utilisent souvent les amandes dans l'élevage pour l'alimentation des porcs. A cela s'ajoute un manque de concasseuse pouvant faciliter les travaux. L'huile de palmiste est aussi utilisée dans la fabrication du savon traditionnel après fermentation. L'huile de palme morte ou issue de la combustion des amandes est utilisée dans la pharmacopée comme frotté pour le massage corporel contre les douleurs musculaires et articulaires, surtout chez les enfants. Elle est également beaucoup utilisée dans la fabrication de savon traditionnel. Les amandes sont aussi utilisées 
comme combustible pour bouillir les noix lors de la production d'huile de palme.

Les résidus de la pulpe après extraction de l'huile de palme, constitués de fibres, sont utilisés à l'état sec pour allumer le feu pendant surtout la saison des pluies. Après égrainage des régimes de palmier à huile, les résidus décomposés sont utilisés comme fertilisant.

\section{La sève}

La sève est recueillie dans des bouteilles placées sous des entailles faites au couteau assez spécialisé. Deux fois par jour, les bouteilles pleines sont remplacées par le récolteur qui grimpe le palmier à l'aide d'une corde passée autour du tronc et de sa taille (Figure 3).

La sève fermentée communément appelée vin de palme est une boisson prisée par la population locale. En période d'exploitation, le vin de palme est consommé chaque jour. Il est, pour la plupart du temps consommé en groupe, donc constitue en effet un réunificateur social. Il est également un produit incontournable dans beaucoup de cérémonies traditionnelles comme les mariages, dot (« manda cabasse »), décès, mais aussi pour les libations. La sève non fermentée était utilisée pour allaiter les nourrissons ayant perdu leur maman. En plus de sa valeur sociale, le vin de palme a une très grande valeur économique. En effet, un exploitant ou un commerçant peut gagner plus de 900 000f CFA avant la fin de la campagne d'exploitation du vin. L'exploitation du vin de palme et la production de l'huile de palme ont joué un rôle très important dans l'émigration des Manjaques. En effet, beaucoup de voyages de migrants ont été financés à travers les revenus provenant de ces activités.

\section{Le tronc ou stipe}

Le stipe ou tronc est beaucoup utilisé dans le domaine de la construction pour la confection des charpentes et le plafonnage des maisons (Figure 1). La coupe pour la construction concerne principalement les vieux sujets de palmiers. Le tronc est utilisé comme bois de chauffe, mais aussi pour la fabrication des ruches et ponts traditionnels, des chaises et des clôtures. Les troncs des palmiers évidés sont parfois utilisés comme des tuyaux dans la réalisation d'ouvrages hydro-agricoles. La poudre issue de la décomposition du stipe est utilisée comme fertilisant.

\section{Les racines}

Les racines du palmier à huile sont utilisées dans la pharmacopée par la population locale. En effet, les racines du palmier à huile sont utilisées pour soigner les maux de dents à partir de la vapeur venant de leur décoction. L'eau tirée de cette décoction, prise comme boisson, permet de soigner la syphilis et les maux de ventre des femmes après accouchement. Les racines servent également à soigner l'obésité. Mélangées à celles de Combretum micranthum, elles soignent l'impuissance sexuelle. La fibre interne de la racine sert à la fabrication des guitares traditionnelles. Pour l'instant, l'exploitation des racines n'est pas si intense à tel point d'avoir des impacts graves sur la survie de l'espèce.

\section{Autres activités réalisées dans la palmeraie}

La palmeraie n'est pas un milieu uniquement réservé à l'exploitation du palmier à huile. Un certain nombre d'activités y sont également menées en dehors de la cueillette. Ces activités sont consignées dans la Figure 5.

Il ressort de l'analyse de la Figure 5 que les activités agricoles dominent largement parmi les multiples activités réalisées dans les parcs à Elaeis guineensis. Il s'agit principalement de l'agriculture pluviale et de l'arboriculture.

Concernant l'agriculture pluviale, les principales spéculations cultivées dans la palmeraie sont représentées par la Figure 6.

L'arachide et le riz de plateau sont les principales spéculations cultivées dans la palmeraie. En plus de celles-ci, viennent le mil, la patate douce et le haricot.

L'interaction entre le palmier à huile et les cultures est jugé positive par les agriculteurs. Ces derniers pensent que le 
palmier à huile augmente la fertilité du sol s'il est élagué. Son ombrage n'a aucun effet négatif sur les cultures.

L'arboriculture concerne principalement les plantations d'anacarde qui ne cessent de gagner du terrain au détriment de la palmeraie.

En dehors des activités agricoles, viennent d'autres comme l'élevage (pâturage) fréquences de citation de à $47 \%$, A celui-ci, s'ajoute la pharmacopée et l'exploitation des produits forestiers ligneux et non ligneux avec une fréquence de citation $27 \%$ pour les deux. D'autres activités comme la production maraîchère, la chasse, et certaines activités culturelles (Fanadu) et cultuelles (fétiches) sont pratiquées dans la palmeraie (Figure 7).

\section{Services écosystémiques fournis par les autres espèces ligneuses}

Les palmeraies de la région de Cacheu renferment une diversité spécifique très importante. En effet, 78 espèces ligneuses ont été enregistrées. Celles-ci sont également exploitées par la population locale pour divers usages. Dans le cadre de la présente étude, les différentes catégories d'usage pris en compte sont l'alimentation humaine, le fourrage, la pharmacopée, le bois d'œuvre, le bois de service et le bois de chauffe. Le mode d'exploitation des différentes espèces ainsi que les valeurs d'usages de chacune sont consignés dans le Tableau 2.

L'Analyse en Composante Principale réalisée a permis de faire une représentation des différentes espèces rencontrées en fonction de leur mode d'exploitation. Sachant que les variables Bois d'œuvre, Bois de service et bois de chauffe ont plus contribué à la formation de l'axe F1et les variables Alimentation humaine et fourrage à celle de l'axe F2, alors la lecture des différentes variables se fera suivant leur axe respectif. Les résultats obtenus sont consignés dans la Figure 8.
L'analyse de la Figure 8 a permis de regrouper les espèces ligneuses rencontrées dans les parcs en différents groupes (groupe $\mathrm{A}, \mathrm{B}$ et $\mathrm{C}$ ) en fonction de leurs usages.

Le groupe A concerne principalement les espèces exploitées pour l'alimentation humaine, la pharmacopée et le fourrage. Il s'agit, entre autres, de Borassus akeassii, Elaeis guineensis, Adansonia digitata, Anacardium occidentale, Parkia biglobosa, Saba senegalensis, Cola cordifolia, Dialium guineense, Landolphia sp, Parinari excelsa, Spondias monbin, Vitex doniana, Uvaria chamae, Sarcocephalus latifolius, Dialium guineense, Neocarya macrophylla.

Le groupe $\mathrm{B}$ concerne les espèces principalement exploitées pour le bois (bois de chauffe, service ou bois de d'œuvre). Il s'agit des espèces comme Pterocarpus erinaceus, Khaya senegalensis, Terminalia macroptera, Zanthoxylum zanthoxyloides, Prosopis africana, Daniellia oliveri, Afzelia Africana, Erythrophleum suaveolens, Carapa procera, Antiaris africana, Oxytenanthera abyssinica, Detarium microcarpum.

Le groupe $\mathrm{C}$ concerne les espèces peu exploitées comme Ficus sp, Fagara zanthoxylum, Macrosphyra longistyla, Alstonia boonei, Cephaelis peduncularis, Combretum paniculatum, Combretum racemosum, Macrosphyra longistyla, Markhamia tomentosa.

$\mathrm{Du}$ point de vue écologique, ces espèces contribuent fortement à la protection des sols notamment dans le maintien de la fertilité. De nombreuses espèces, principalement de la sous-famille des Faboideae ont la particularité de puiser l'azote à la fois dans le sol et l'air. Elles se caractérisent par une activité symbiotique de fixation de l'azote atmosphérique grâce aux bactéries présentes dans leurs nodosités. L'agriculture exploite cette particularité naturelle des Faboideae à travers l'alternance et l'association avec d'autres cultures. 
Tableau 1 : Parties du palmier à huile exploitées et les différents usages.

\begin{tabular}{|c|c|c|c|}
\hline Partie & Sous-partie & Usage & FC $(\%)$ \\
\hline \multirow{12}{*}{ Feuille } & \multirow{3}{*}{ Feuille entière } & Clôture & 93 \\
\hline & & Couverture des régimes & 7 \\
\hline & & Piège de poisson & 7 \\
\hline & \multirow{2}{*}{ Foliole } & Fumier & 33 \\
\hline & & Entonnoir & 60 \\
\hline & \multirow{6}{*}{ Nervure principale } & Ceinture & 60 \\
\hline & & Palissade & 7 \\
\hline & & Lit & 13 \\
\hline & & Corde & 40 \\
\hline & & Panier de stockage de sel & 40 \\
\hline & & Plafond & 33 \\
\hline & Nervure secondaire & Balais & 100 \\
\hline \multirow{4}{*}{ Fleur } & \multirow{4}{*}{ Fleur } & Récolte de miel & 33 \\
\hline & & insectifuge & 80 \\
\hline & & Savon & 40 \\
\hline & & Fumier & 27 \\
\hline \multirow{9}{*}{ Régime } & \multirow{4}{*}{ Amande } & Savon & 93 \\
\hline & & Alimentation des porcs & 47 \\
\hline & & Huile palmiste & 27 \\
\hline & & Pommade de massage & 7 \\
\hline & Grappe et pulpe & Fumier & 20 \\
\hline & \multirow{4}{*}{ Pulpe ou mésocarpe, } & Huile de palme & 93 \\
\hline & & $\begin{array}{l}\text { Sauces de palmiste (Thieben en } \\
\text { créole) }\end{array}$ & 73 \\
\hline & & Allumage du feu & 60 \\
\hline & & Eclairage (Kandjirba) & 7 \\
\hline \multirow{3}{*}{ Sève } & \multirow{3}{*}{$\begin{array}{l}\text { Sève fermentée ou vin } \\
\text { de palme }\end{array}$} & Consommation & 67 \\
\hline & & Cérémonie & 73 \\
\hline & & Libation & 47 \\
\hline \multirow{7}{*}{ Stipe } & \multirow{7}{*}{ Stipe } & Construction & 100 \\
\hline & & Ruche traditionnelle & 7 \\
\hline & & Clôture & 20 \\
\hline & & Chaise & 7 \\
\hline & & Pont traditionnel & 7 \\
\hline & & Fumier & 7 \\
\hline & & Bois de chauffe & 13 \\
\hline \multirow{3}{*}{ Racine } & Racine entière & Pharmacopée & 73 \\
\hline & \multirow{2}{*}{ Fibre interne } & Corde & 7 \\
\hline & & Guitare & 60 \\
\hline
\end{tabular}



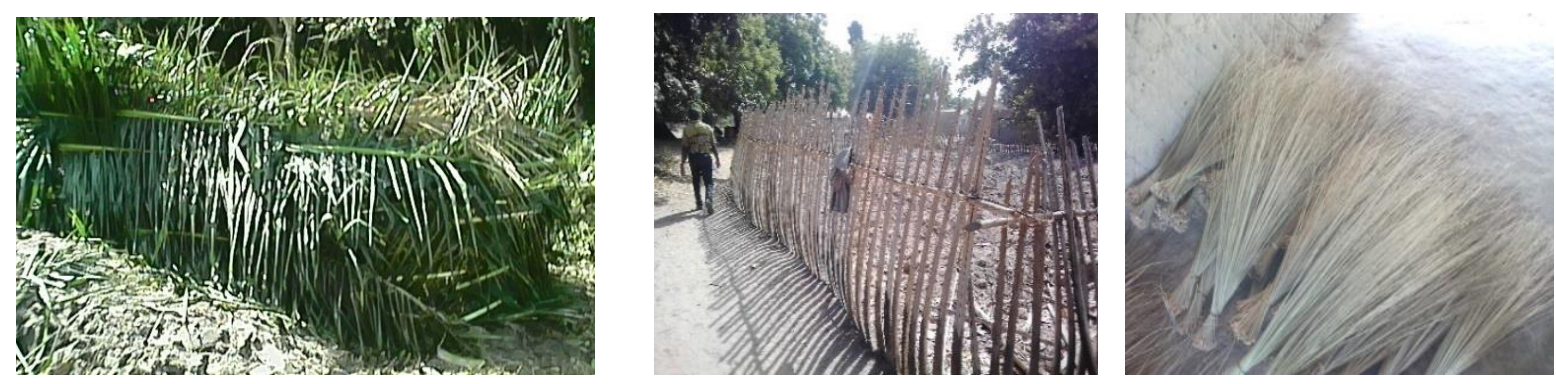

Figure 2 : Quelques usages faites à partir des feuilles de Elaeis guineensis.
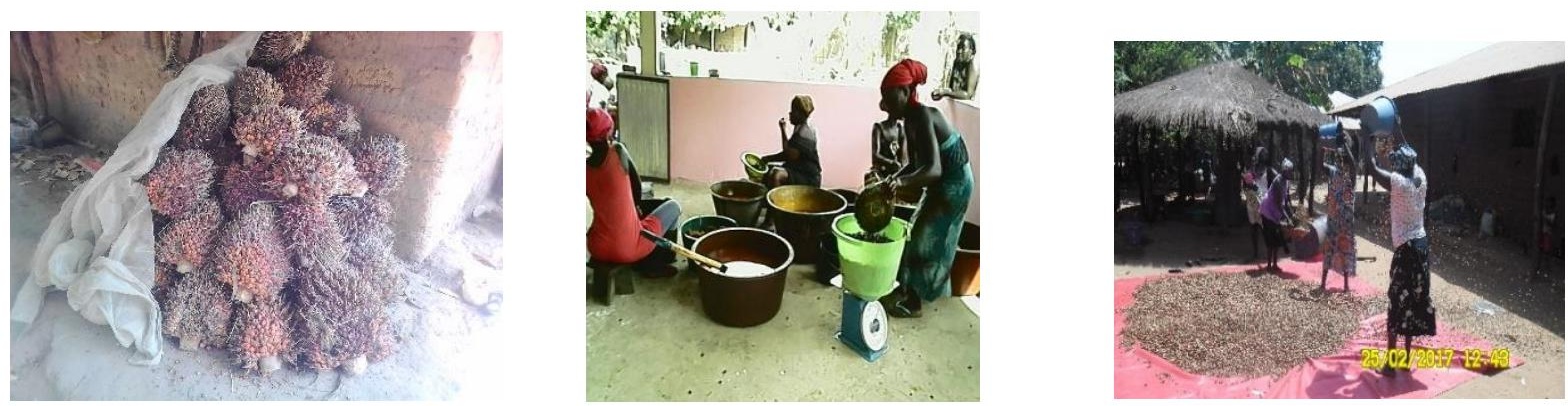

Figure 3 : Utilisation des régimes de Elaeis guineensis.
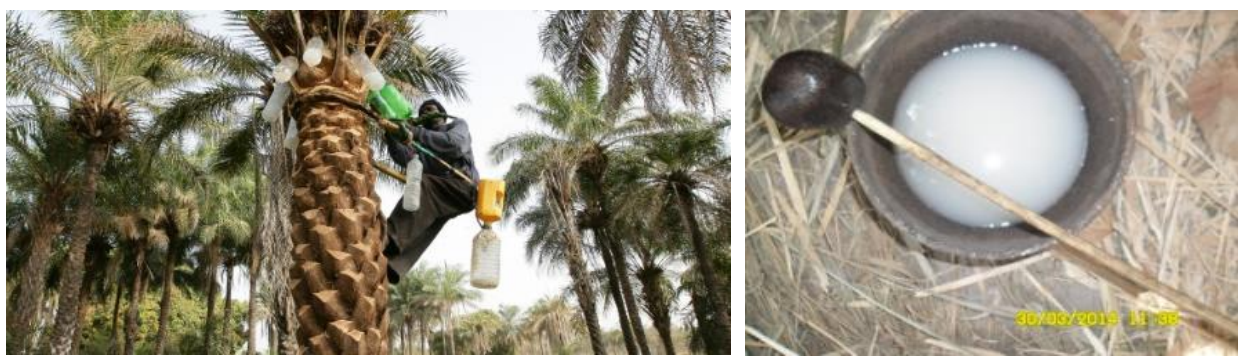

Figure 4 : Technique de récolte du vin de palme.
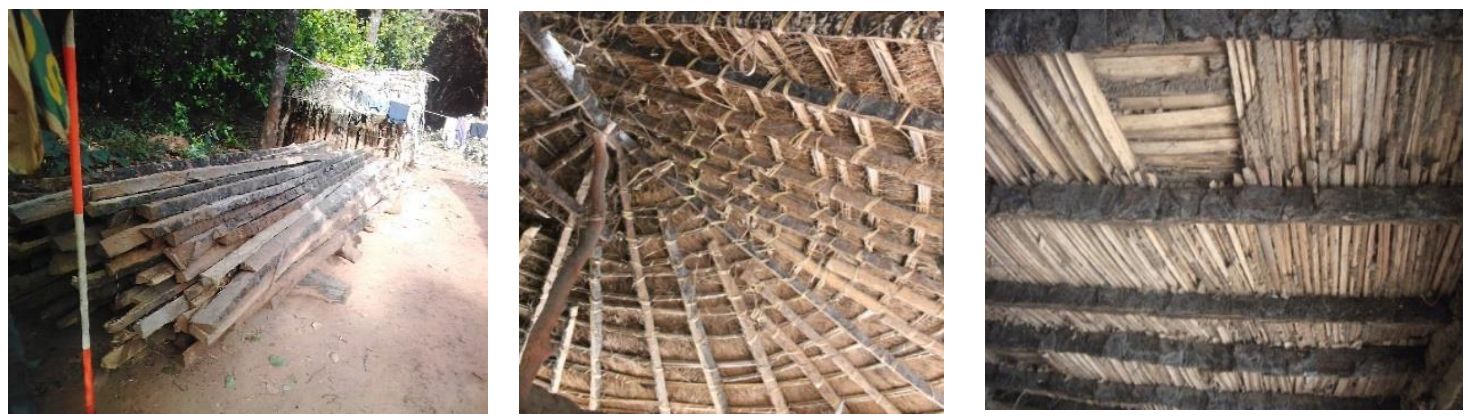

Figure 5 : Utilisation des stipes de Elaeis guineensis dans la construction. 


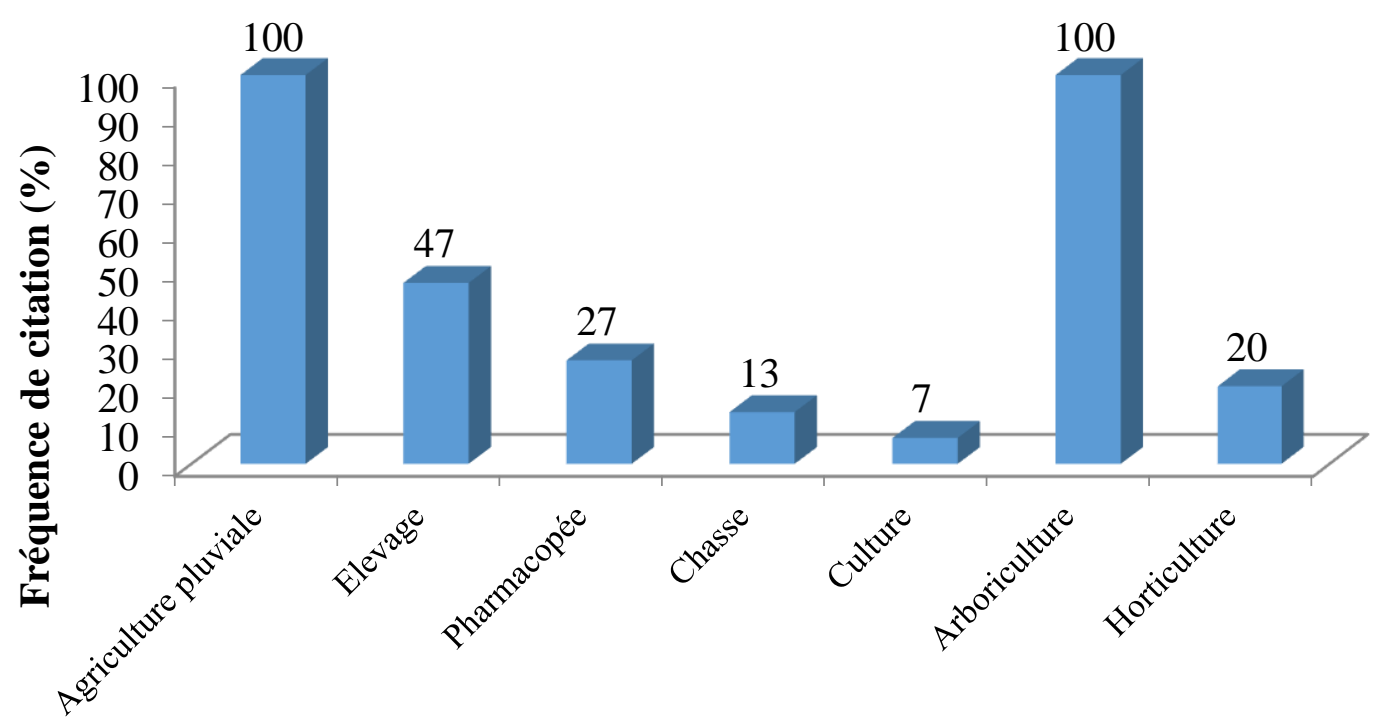

Figure 6 : Autres activités réalisées dans la palmeraie.

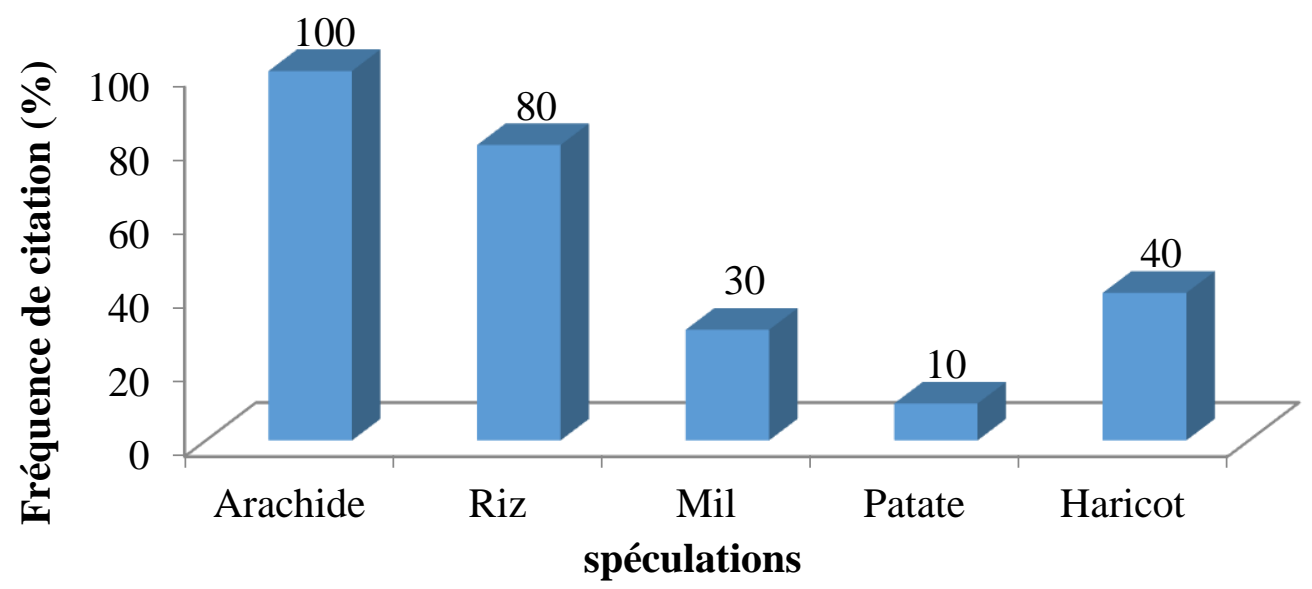

Figure 7: Cultures associées au palmier à huile.
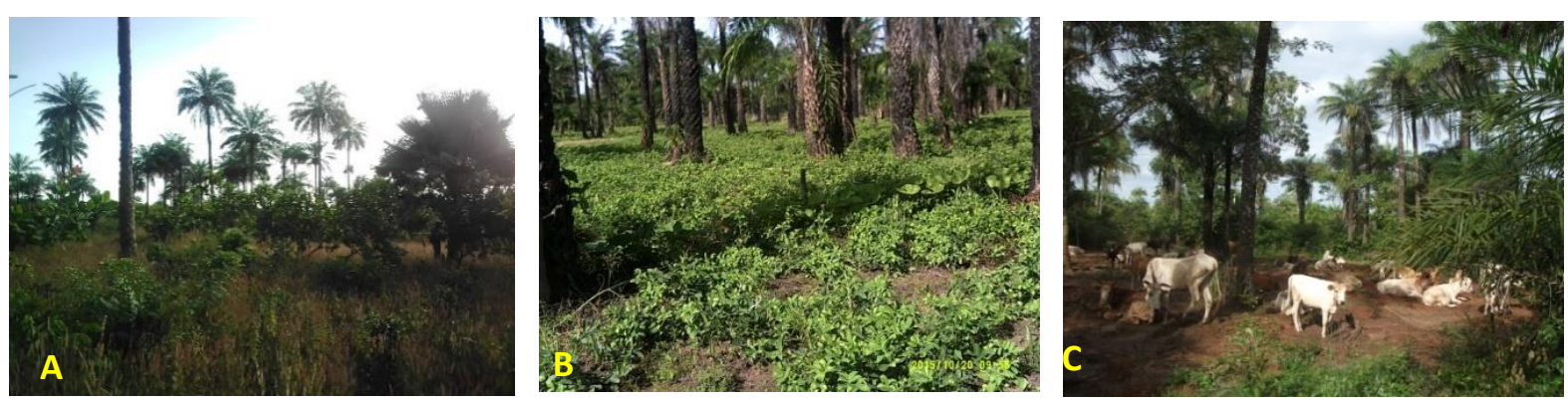

Figure 8 : Quelques activités réalisées dans les parcs à Elaeis guineensis $(\mathrm{A}=$ Arboriculture $\mathrm{B}=$ Agriculture pluviale, $\mathrm{C}=$ Elevage). 
Tableau 2 : Services écosystémiques fournis par les autres espèces de la palmeraie.

\begin{tabular}{|c|c|c|c|c|c|c|}
\hline \multirow{2}{*}{ Espèces } & \multirow[b]{2}{*}{ Famille } & \multicolumn{3}{|l|}{ Noms vernaculaires } & \multirow[b]{2}{*}{ Usage } & \multirow{2}{*}{$\mathbf{V u}$} \\
\hline & & Manjaque & Créole & Balanta & & \\
\hline Adansonia digitata $\mathrm{L}$. & Bombacaceae & Bu rungal & Cabacera & Blaté & $\mathrm{Al}, \mathrm{Fo}, \mathrm{Pha}, \mathrm{Bs}, \mathrm{Bc}$ & 83 \\
\hline Afzelia Africana Smith ex pers. & Fabaceae & Bu Biacar & Po di conta & Pegré & Fo, Pha, Bo, Bs, Bc & 83 \\
\hline Albizia adianthifolia (Schumach.) & Fabaceae & Bilélbring & Foroba de lala & Pangza & Fo, Pha, Bo, Bs, Bc & 83 \\
\hline Albizia lebbeck (L.) Benth. & Fabaceae & & & & Fo, Bo, Bs, Bc & 67 \\
\hline Albizia zygia (DC.) J.F. Macbr. & Fabaceae & Biangni & Po de rayo & N'grobé & $\mathrm{Fo}, \mathrm{Bo}, \mathrm{Bs}, \mathrm{Bc}$ & 67 \\
\hline Alchornea cordifolia Schumach. Et Thonn. & Euphorbiaceae & Bu gongue & Arcu & Pos & Fo, Pha, Bs, Bc & 67 \\
\hline Allophyllus africanus P.Beauv. & Sapindaceae & Kawoybayafa & Podimosca & Mbudri/ Manao & $\mathrm{Al}, \mathrm{Fo}, \mathrm{Pha}, \mathrm{Bs}, \mathrm{Bc}$ & 83 \\
\hline Alstonia boonei De Wild & Apocynaceae & Bietéte & & & Fo, Pha & 33 \\
\hline Anacardium occidentale $\mathrm{L}$. & Anacardiaceae & Bu kadju & Kadju & kaju & $\mathrm{Al}, \mathrm{Fo}, \mathrm{Pha}, \mathrm{Bs}, \mathrm{Bc}$ & 83 \\
\hline Annona senegalensis Pers. & Annonaceae & Binataro & Manduko di futicero & Fiam/ Bodiori & $\mathrm{Al}, \mathrm{Fo}, \mathrm{Pha}, \mathrm{Bs}, \mathrm{Bc}$ & 83 \\
\hline Anthocleista nobilis G. Don & Gentianaceae & Beu pal/ Beu bak ughil & Tabaco di lobu & Bpufa & Fo, Pha,Bs & 50 \\
\hline Anthostema senegalense A. Juss. & Euphorbiaceae & Beutathié & Po di liti/ Bignalé & Bruni/ Pfuni & Fo, Bs, Bc & 50 \\
\hline Antiaris africana Lesch. & Moraceae & Beu Kancal & & & Fo, Pha, Bo, Bs, Bc & 83 \\
\hline Azadiracht aindica Hutch. & Meliaceae & Bu kassia & Nivaquina & Tapfélé & Fo, Pha, Bo, Bs, Bc & 83 \\
\hline Bombax costatum Pellegr. \&Vuillet & Malvaceae & Blor lor & & Kila kila & $\mathrm{Al}, \mathrm{Fo}, \mathrm{Pha}, \mathrm{Bo}, \mathrm{Bs}, \mathrm{Bc}$ & 100 \\
\hline Borassus akeassii Mart. & Arecaceae & Beu bel & Sibi & N'kotne & $\mathrm{Al}, \mathrm{Fo}, \mathrm{Pha}, \mathrm{Bo}, \mathrm{Bs}, \mathrm{Bc}$ & 100 \\
\hline Carapa procera DC. & Meliaceae & Bok & Po di sitimalgos & Pkagné & Fo, Pha, Bo, Bs, Bc & 83 \\
\hline
\end{tabular}


B. SAGNA et al. / Int. J. Biol. Chem. Sci. 13(7): 3289-3306, 2019

\begin{tabular}{|c|c|c|c|c|c|c|}
\hline Cassia alata (L.) Roxb & Fabaceae & Bi gnélé & Padia santé & & Pha, Bc & 33 \\
\hline Cassia sieberiana Del. & Fabaceae & Beu tambe & Canafistra & Sogho & Fo, Pha, Bo, Bs, Bc & 83 \\
\hline Ceiba pentendra L. & Bombacaceae & Puntia & Polon & Bushahé & $\mathrm{Al}, \mathrm{Fo}, \mathrm{Pha}, \mathrm{Bo}, \mathrm{Bs}, \mathrm{Bc}$ & 100 \\
\hline Cephaelis peduncularis (Schnell) & Rubiaceae & Beu bungué & & Bdigubitche & Fo, Pha & 33 \\
\hline Cnestis ferruginea DC. & Connaraceae & Butonkubus & & & Pha, Bs, Bc & 50 \\
\hline Cola cordifolia (Cav.) & Malvaceae & Bu ntaba & N'taba & Mbué & $\mathrm{Al}, \mathrm{Fo}, \mathrm{Pha}, \mathrm{Bo}, \mathrm{Bs}, \mathrm{Bc}$ & 100 \\
\hline Combretum micranthum G. Don & Combretaceae & Buk & Buk & Sanglan & $\mathrm{Al}, \mathrm{Pha}, \mathrm{Bs}, \mathrm{Bc}$ & 67 \\
\hline Combretum paniculatum (vent,) & Combretaceae & & & & Fo, Pha, Bs, Bc & 67 \\
\hline Combretum racemosum $\mathrm{P}$. Beauv. & Combretaceae & & & & Fo, Pha, Bs, Bc & 67 \\
\hline Daniellia oliveri wild & Fabaceae & & & Bobdé & Fo, Pha, Bo, Bs, Bc & 83 \\
\hline Detarium microcarpum (J. F. Gmelin) & Fabaceae & Bobuar & Mamboré & Plund & Pha, Bo, Bs, Bc & 67 \\
\hline Dialium guineense (Willd) & Fabaceae & Bubuy & Veludu & Mbuyu & $\mathrm{Al}, \mathrm{Fo}, \mathrm{Pha}, \mathrm{Bo}, \mathrm{Bs}, \mathrm{Bc}$ & 100 \\
\hline Dichrostachys cinerea (Wight et Arn.) & Fabaceae & Bintheumplibignel & Pé di fididapreto & Yué/ N'dioh & $\mathrm{Fo}, \mathrm{Bs}, \mathrm{Bc}$ & 50 \\
\hline Ekebergia senegalensis A. Juss. & Meliaceae & & & & Fo, Pha, Bs, Bc & 67 \\
\hline Elaeis guineensis (Jacq.) & Arecaceae & Bi thiam & Palmera & Kéngh & Al, Fo, Pha, Bo, Bs, Bc & 100 \\
\hline Erythrina senegalensis L. & Fabaceae & Bithiante & Po di ndoli/ Bissaka & Nfisi & Fo, Pha,Bs, Bc & 67 \\
\hline Erythrophleum guineense G. Don & Fabaceae & Betabeu & Manconi & Ptome & Fo, Pha, Bs, Bc & 67 \\
\hline Erythrophleum suaveolens (Guill. \&Perr.) & Fabaceae & & & & Pha, Bo, Bs, Bc & 67 \\
\hline Fagara zanthoxylum (L.) Sarg. & Rutaceae & Bi thiel & Ghugna di onsa & Kombé tonka & Fo, Pha, Bs & 50 \\
\hline Ficus elastica (Roxb. ex Hornem.) & Moraceae & Bu pokbuyente & & & Fo, Pha, Bs, Bc & 67 \\
\hline Ficus exasperata (Vahl) & Moraceae & Bu pok & & & Fo, Pha, Bs, Bc & 67 \\
\hline Ficus sur (Forssk.) & Moraceae & Bu pok & & & Fo, Pha,Bs, Bc & 67 \\
\hline
\end{tabular}


B. SAGNA et al. / Int. J. Biol. Chem. Sci. 13(7): 3289-3306, 2019

\begin{tabular}{|c|c|c|c|c|c|c|}
\hline Ficus sycomorus L. & Moraceae & $\mathrm{Bu}$ pok & & & Al, Fo, Pha, Bs, Bc & 83 \\
\hline Ficus vogelii (Miq.) & Moraceae & Bu kungul & & & Fo, Pha & 33 \\
\hline Guiera senegalensis J.F. Gmel & Combretaceae & Bi Nthinte & Badodos & Biozet & Pha, Bs, Bc & 50 \\
\hline Hibiscus sterculiifolius (Guill. \&Perr.) & Malvaceae & Bu liaba & Pé di nancignu & & Fo, Pha, Bs, Bc & 67 \\
\hline Holarrhena floribunda (G.Don) & Apocynaceae & Biététe & Po di cudier & Brikpath/ Rupitcha & Fo, Pha, Bo, Bs, Bc & 83 \\
\hline Khaya senegalensis (Desr.) & Meliaceae & Bintia & Bissilon & Ptagmi & Fo, Pha, Bo, Bs, Bc & 83 \\
\hline landolphia dulcis (Sabine ex G.Don) & Apocynaceae & Bu kubar & Pé di Mancubar & Blindé & Al, Fo, Pha & 50 \\
\hline Landolphia heudelotii (P. Beauv.) & Apocynaceae & Beutah & Folé & Psubé & Al, Fo, Pha, Bs & 67 \\
\hline Landolphia owariensis(P.Beauv.) & Apocynaceae & Bu kute & & & Al, Fo, Pha, Bs & 67 \\
\hline Lophir alanceolata Van Tiegh. ex Keay & Ochnaceae & & & & $\mathrm{Pha}, \mathrm{Bs}, \mathrm{Bc}$ & 50 \\
\hline Macrosphyr alongistyla (DC.) & Rubiaceae & Bu badiuthiak & Tépolo & Psanglablate & Fo, Pha & 33 \\
\hline Malancantha alnifolia (Bak.) Pierre & Sapotaceae & Binthian & & & Fo, Pha,Bs, Bc & 67 \\
\hline Mangifera indica (L.) & Anacardiaceae & Beumangué & Pé di mango & Mango & $\mathrm{Al}, \mathrm{Fo}, \mathrm{Pha}, \mathrm{Bs}, \mathrm{Bc}$ & 83 \\
\hline Markhamia tomentosa (Benth,) & Bignoniaceae & & & Nkinguiteblante & Pha, Bs, Bc & 50 \\
\hline Mimosa pigra $\mathrm{L}$. & Mimosaceae & Bu gnéréweute & & & Pha, Bs, Bc & 50 \\
\hline Morinda geminata (DC.) & Rubiaceae & Bu kuy & Bulonguedjiba & Fughankila & Pha, Bs, Bc & 50 \\
\hline Neocarya macrophylla (Sabine) & Chrysobalanaceae & Bi naw & Tambacumba & Npking/ Flékhet & Al, Fo, Pha, Bs, Bc & 83 \\
\hline Newbouldia leavis (P.Beauv.) & Bignoniaceae & & Manduko di futiséro & Nkinguite & Pha, Bs, Bc & 50 \\
\hline Oxytenanthera abyssinica (A. Rich.) Munro & Poaceae & Pu Diame & Kana di Bambu & Psuka & Pha, Bo, Bs, Bc & 67 \\
\hline Parinari excelsa (Sabine) & Chrysobalanaceae & Bu thialang & Mampatas & Pilé & Al, Fo, Pha, Bs, Bc & 83 \\
\hline Parkia biglobosa (Jacq.) & Fabaceae & Beulèl & Foroba & Ghanté/Mouhanfé & $\mathrm{Al}, \mathrm{Fo}, \mathrm{Pha}, \mathrm{Bs}, \mathrm{Bc}$ & 83 \\
\hline Piliostigma thonningii (Schum.) & Fabaceae & Beutunkal & Po di pé di baka & M'bogha/ Ponka & Fo, Pha, Bo, Bs, Bc & 83 \\
\hline
\end{tabular}


B. SAGNA et al. / Int. J. Biol. Chem. Sci. 13(7): 3289-3306, 2019

\begin{tabular}{|c|c|c|c|c|c|c|}
\hline Prosopis africana (Guill. \&Perr.) & Fabaceae & Bi dji & Po di carbon & Tintir & Fo, Pha, Bo, Bs, Bc & 83 \\
\hline Pterocarpus erinaceus (Poir.) & Fabaceae & Biliadji & Po di sangui & Psila & Fo, Pha, Bo, Bs, Bc & 83 \\
\hline Raphia vinifera A. Chev. & Arecaceae & Peu tate & Tara & Dar & $\mathrm{Al}, \mathrm{Fo}, \mathrm{Pha}, \mathrm{Bs}$ & 67 \\
\hline Saba senegalensis (A. DC.) & Apocynaceae & Bu koncar & Folélifanté & Mbegdé & $\mathrm{Al}, \mathrm{Fo}, \mathrm{Pha}, \mathrm{Bs}, \mathrm{Bc}$ & 83 \\
\hline Sarcocephalus latifolius (Sm.) & Rubiaceae & Beunaouthiata & Madronha & Té togodo & $\mathrm{Al}, \mathrm{Fo}, \mathrm{Pha}, \mathrm{Bs}, \mathrm{Bc}$ & 83 \\
\hline Sorindeia juglandifolia (A. Rich.) & Anacardiaceae & & & Péigré & Fo, Pha, Bs, Bc & 50 \\
\hline Spondias monbin L. & Anacardiaceae & Bi pialam & Mandipli & Psag & $\mathrm{Al}, \mathrm{Fo}, \mathrm{Pha}, \mathrm{Bs}, \mathrm{Bc}$ & 83 \\
\hline Strychnos spinosa Lam. & Logaminaceae & Mbimblinpol & Limon di matu & & Fo, Pha, Bs, Bc & 67 \\
\hline Tephrosia purperea Pers. & Fabaceae & Bu tundji & & Mpasha & Pha, Bc & 33 \\
\hline Terminalia macroptera (Guill. \&Perr.) & Combretaceae & Beu talé & Masiti & Fadi & Fo, Pha, Bo, Bs, Bc & 83 \\
\hline Uvaria chamae (P.Beauv.) & Annonaceae & Bu ngundian & Banana santhiu & Nfenté néné & $\mathrm{Al}, \mathrm{Fo}, \mathrm{Pha}, \mathrm{Bs}, \mathrm{Bc}$ & 83 \\
\hline Vitex doniana $(\mathrm{L})$. & Lamiaceae & Ngoua & Azeitona & Muni & $\mathrm{Al}, \mathrm{Fo}, \mathrm{Pha}, \mathrm{Bs}, \mathrm{Bc}$ & 83 \\
\hline Voacanga africana (Stapf ex Scott-Elliot) & Apocynaceae & Bu fanté & Po di buracha & Blakhakhé/Boghé & Fo, Pha, Bo, Bs, Bc & 83 \\
\hline Zanthoxylum zanthoxyloides (Lam.) & Rutaceae & Bu tonk & Taw-taw/ bi tŏnk & Paw-paw & Fo, Pha, Bs & 50 \\
\hline
\end{tabular}




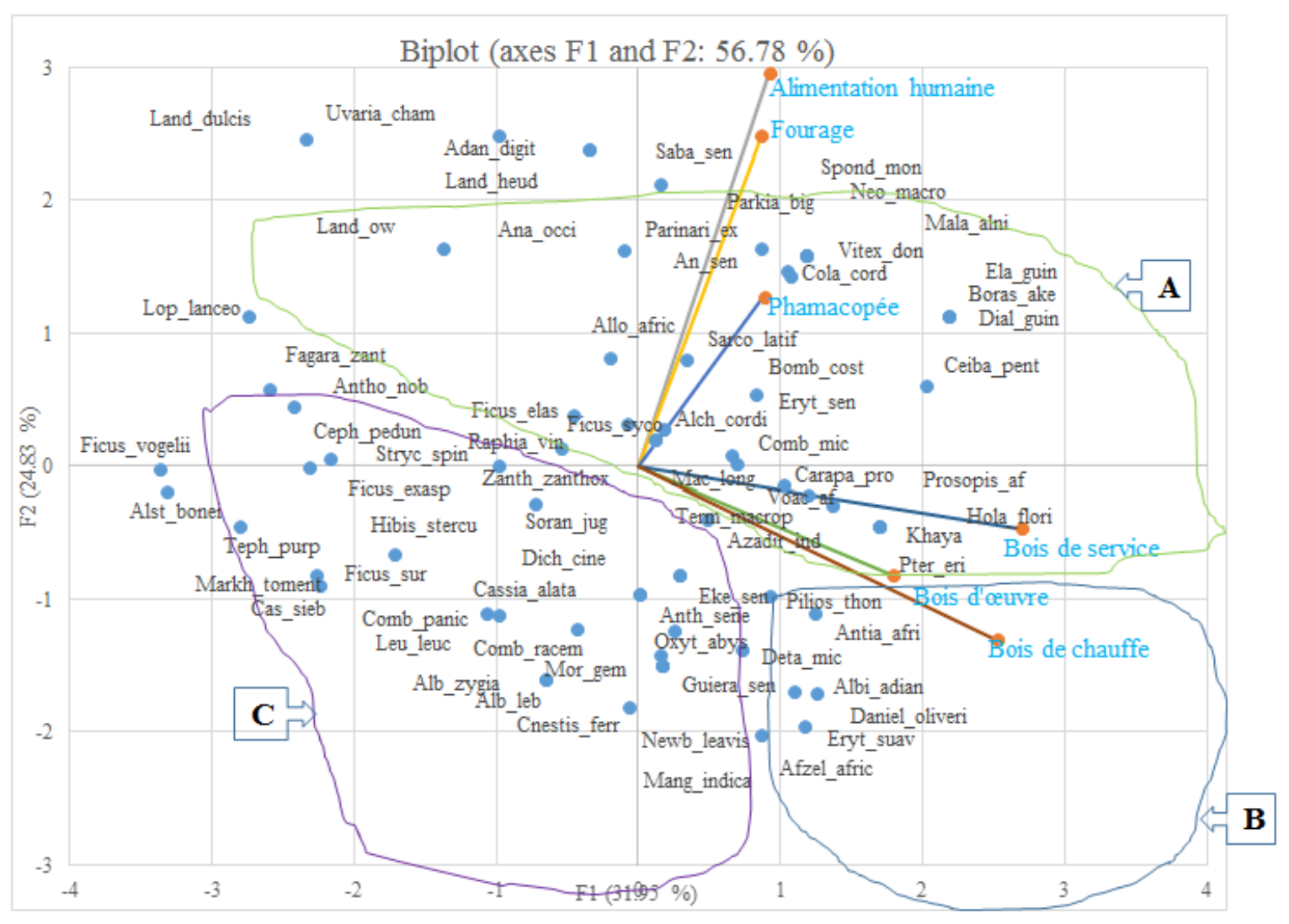

Figure 9 : Répartition des espèces ligneuses en fonction des catégories d'usage.

\section{DISCUSSION}

Les parcs à Elaeis guineensis ou palmeraies constituent des écosystèmes à importance socioéconomique inestimable. Si l'on considère d'abord Elaeis guineensis qui est l'espèce caractéristique de ces systèmes de production, son importance est dans plusieurs domaines :

Dans le domaine de l'alimentation humaine: les produits comme l'huile de palme, la soupe de palmiste (Thébin), le vin de palme, etc. contribuent beaucoup à l'alimentation de la population locale. Ce résultat corrobore celui d'Angerand (2007) qui affirme que ces huiles sont à la base de nombreux régimes alimentaires, notamment en Afrique. L'huile de palme est riche en vitamine $\mathrm{A}$ et $\mathrm{E}$. La vitamine $\mathrm{A}$ joue un rôle important dans la vision, notamment au chapitre de l'adaptation de l'œil à l'obscurité.
Elle participe aussi à la croissance des os, à la reproduction et à la régulation du système immunitaire. Elle contribue à la santé de la peau et des muqueuses (yeux, voies respiratoires et urinaires, intestins), qui constituent notre première ligne de défense contre les bactéries et les virus. La vitamine E, quant à elle, a un effet protecteur particulièrement important vis-à-vis des cellules de l'organisme. Elle joue un rôle important dans les mécanismes de la procréation et intervient dans la synthèse des globules rouges.

La boisson à base de sève de palmier ou vin de palme contient également de la vitamine B1, B2, B3 et B6. Toutes les vitamines $\mathrm{B}$ aident l'organisme à transformer les aliments (glucides) en carburant (glucose), qui sert à produire de l'énergie. En plus de produire de l'énergie pour le corps, la 
riboflavine agit comme un antioxydant, combattant les particules nocives dans le corps appelées radicaux libres pouvant endommager les cellules et l'ADN et contribuer au processus de vieillissement (Girardin et al., 2002). Le vin de palme présente de nombreux avantages pour la santé, en particulier lorsqu'il est consommé frais et non fermenté. Il est également riche en acides aminés, potassium, magnésium, zinc et fer. Contrairement à Niang (2014) selon qui, les techniques d'exploitation du vin en Basse Casamance sont des pratiques durables qui se font depuis des générations et qui contribuent à assainir les populations de palmiers à huile, ce qui limite la prolifération des insectes ravageurs, aujourd'hui pour des raisons économiques, on note dans la région de Cacheu la prolifération de techniques de récolte permettant une sortie massive de sève qui engendre souvent de graves conséquences sur les palmiers. Il s'agit, entre autres, de l'exploitation sans arrêt sur le même sujet (+15 jours ou chaque année), la fixation d'un grande pointe, après élagage, sur le tronc, la récolte le vin à partir des fleurs, les coups de marteau tout autour du stipe après élagage des feuilles pour le ramollir et intensifier la sortie de vin, mélange de produit comme ceux fait à base de feuilles de Moringa oleifera et de Adansonia digitata broyées pour créer une chaleur autour du tronc afin d'accélérer la sortie de la sève, etc. D'ailleurs c'est ce qui a poussé au Grdr à penser, à travers des enquêtes réalisées en 2015, que les mauvaises techniques d'exploitation de vin constituent une des causes de la baisse de production des palmiers. Ce phénomène est surtout encouragé, selon les populations l'implantation saisonnière de récolteurs étrangers.

La consommation des produits du palmier à huile a tendance à augmenter chez les familles productrices du fait de la prise de conscience progressive des bienfaits la consommation des produits locaux comme l'huile de palme sur la santé humain (Manolescu, 2009).

$\mathrm{Du}$ point de vue écologique, les palmeraies contribuent à l'enrichissement du sol (Sagna, 2016 ; Sagna 2017 et Camara et al. (2018). En effet, le palmier à huile augmente la fertilité des sols et son effet d'ombrage n'est pas significatif s'il est élagué comme constaté par Ngom et al. (2015), Sagna (2016) et Sagna (2017). Le même constat a également été fait par Akouehou et al. (2013) qui affirment que dans le département de l'Atlantique (Sud du Bénin), les raisons qui ont motivé les producteurs à maintenir une densité forte de palmiers à huile avec des cultures annuelles sont liées à sa capacité à remonter la fertilité des terres.

Pour la construction de la plupart des maisons, le palmier est une espèce incontournable dans la confection des plafonds et charpentes. Ce même constat a été fait par certains auteurs tels que CIRAD (2008) et Carrere (2010) selon qui, le palmier à huile fournit des matériaux de construction aux populations.

Sur le plan économique, les produits du palmier notamment le vin et l'huile de palme procurent aux exploitants beaucoup de ressources. Ce résultat est en accord avec celui de Ofosu-Budu et Sarpong (2013) qui affirment que les palmeraies et les huileries, ainsi que l'ensemble des services liés à la production, ont contribué et continuent de nos jours à contribuer au développement des économies locales en Guinée Forestière et en Basse Guinée.

Sur le plan cultuel et culturel, le vin est incontournable dans certaines cérémonies traditionnelles. C'est ce qui conduit Yombouno (2014) à affirmer que le palmier à huile est considéré comme source d'économie traditionnelle et comporte des valeurs socioculturelles importantes.

Dans le domaine de la santé, certaines parties du palmier à huile sont utilisées pour soigner des maladies. Cette même remarque a été faite par beaucoup d'auteurs comme Arbonnier (2000), CIRAD (2008), Diatta et Sagna (2013), Sagna (2016) et Sagna (2017) selon qui, le palmier à huile est beaucoup exploité pour la médecine traditionnelle.

Dans l'industrie locale, certains produits du palmier à huile sont utilisés pour produire du savon, frotté, etc. Ce résultat est 
en accord avec Ofosu-Budu et Sarpong (2013) qui ont montré que le palmier à huile, un oléagineux essentiel, fournit beaucoup de produits, à la fois pour la consommation domestique et comme matière première pour le secteur de l'industrie.

Comme constaté par Diédhiou et al. (2014), Camara (2018) et Badiane et al. (2019) au niveau des parcs au Sénégal très riches en biodiversité, il existe dans les palmeraies de la région de Cacheu une longue liste d'espèces accompagnatrices de Elaeis guinnensis. L'exploitation des espèces forestières contribue fortement à améliorer les conditions de subsistances de la population locale notamment dans le domaine de l'alimentation, de l'économie et du bien-être social. En effet, $24 \%$ des espèces de la palmeraie appartiennent à la famille des Fabaceae. Celle-ci représente, sur le plan économique, la deuxième famille en importance après les Poaceae et constituent une source de protéines végétales très appréciable pour l'alimentation humaine (Graham et Vance, 2003). C'est dans cet ordre d'idée que Goudiaby (2013), Gomis (2014), Ngom (2014) et Sagna (2016), ont affirmé que les parcs agroforestiers contribuent fortement à la subsistance de la population locale.

\section{Conclusion}

La région de Cacheu couvre d'importantes formations naturelles de palmeraies. Ces formations, autrement appelées parcs agroforestiers à Elaeis guineensis, renferment une flore ligneuse diversifiée. Cette étude qui s'était fixée comme objectif de contribuer à l'évaluation de l'importance socioéconomique de ces parcs a permis de comprendre que le palmier qui est l'espèce caractéristique de ces systèmes est exploité sur toutes ses composantes pour divers usages contribuant ainsi à l'amélioration du bien-être social de la population. Les autres espèces ont aussi une importance capitale dans la vie de la population locale. Au vu de l'importance de ces parcs dans le quotidien des populations de la région, il est impératif d'assurer la pérennisation de ces systèmes de production à travers la valorisation et l'exploitation durable des palmeraies naturelles de la région, la garantie du renouvellement des parcs par des techniques de régénération naturelle assistée (RNA), le respect d'un écartement raisonnable lors de l'établissement de nouvelles plantations d'Anacardium occidentale afin de permettre l'association avec d'autre espèces notamment Elaeis guineensis.

\section{CONFLIT INTERETS}

Les auteurs de cet article déclarent ne pas avoir de conflit d'intérêts.

\section{CONTRIBUTIONS DES AUTEURS}

BSA conçu l'étude et les auteurs tels que DN, MAAD et BC ont lu et apporté des suggestions pour l'amélioration du projet de recherche. YLC, chargé de la RA au Grdr a examiné de manière critique le projet. BS a effectué les travaux de terrain et organisé l'atelier de restitution avec l'appui de ASM. Tous les auteurs ont lu et apporté leurs contributions à la version finale du manuscrit.

\section{REMERCIEMENTS}

Nous tenons à remercier également les institutions telles que le Grdr, le Laboratoire d'Écologie végétale de l'Université Cheikh Anta Diop de Dakar, le Laboratoire d'Agroforesterie et d'Ecologie de l'Université Assane SECK de Ziguinchor et l'Inspection régionale des Eaux et Forêts, Chasse et Conservation des Sols de Ziguinchor pour leur collaboration dans la réalisation de l'étude. Nous remercions également les populations des villages choisis pour l'étude pour l'accueil, leur disponibilité, leur collaboration durant les enquêtes.

Notre gratitude à Ndiouga Sarr BADJI et René Joachim MANGA, élèves au Centre National de Formation des Techniciens de Eaux et Forêts pour leur soutien technique lors de la réalisation des travaux de terrain.

\section{REFERENCES}

Akouehou GS, Assogba DO, Houndonougbo A, Sinsin AB. 2013. Diversité floristique, sécurisation foncière et gestion des systèmes agroforestiers à 
palmier à huile (Elaeis guineensis) en zones périurbaines et rurales du Département de l'Atlantique au Sud du Bénin. Int. J. Biol. Chem. Sci., 7(3): 1180-1189. DOI: 10.4314/ijbcs.v7i3.24 Arvanitis Y. 2014. Ressources naturelles en Guinée-Bissau : partir du bon pied. Plan stratégique et opérationnel 20152020, $56 \mathrm{p}$. https://www.afdb.org/fr/blogs/measuri ng-the-pulse-of-economic-

transformation-in-west

africa/post/natural-resources-inguinea-bissau-getting-it-right-fromthe-start-13630/.

Badiane M, camara B, Ngom D, Diedhiou M AA. 2019. Perception communautaire des parcs agroforestiers traditionnels à Faidherbia albida (Del.) Chev. en Basse Casamance, Sénégal. Afrique Science, 15(1): 214-226. http://www.afriquescience.net.

Banjai BB. 2009. Quatrième Rapport National sur la Diversité Biologique, 99 pages.

Bonkoungou EG, Ayuk ET, Zoungrana I. 1997. Les parcs agroforestiers des zones semi-arides d'Afrique de l'Ouest. Actes du séminaire international (25-27/10/1993), Ouagadougou, Burkina Faso. 225 p.

Buffiere M. 1984. La palmeraie naturelle dans le département d'Oussouye: Les revenus tirés de son exploitation et les possibilités de son amélioration. Rapport de stage, SOMIVAC/BBEP.

Carrere R. 2010. Le palmier à huile en Afrique : le passé, le présent et le futur. Mouvement mondial pour les Forêts tropicales. Collection du WRM sur les plantations $\mathrm{N}^{\circ} 15$.

Bonnet P, Arbonnier M, Grard P. 2008. Versailles : Ed. Quae, 1 Cd-Rom.

Camara B. 2018. Caractérisation agroécologique et socio-économique des parcs agroforestiers à Elaeis guineensis Jacq. Et Faidherbia albida (Del.) Chev. et leurs influences sur la productivité du riz pluvial en basse Casamance (Sénégal). Thèse unique,
ED/STI/ Université Assane SECK /Ziguinchor Sénégal, 153 p.

Camara B, Gosme M, Ngom D, Gomis ZD, Badji M, Sanogo D, Dupraz C. 2018. Ecological characterization and evolution of Elaeis guineensis Jacq. traditional parklands in Lower Casamance (Senegal). Agroforest Syst., 93(4): $\quad 1251-126 . \quad$ DOI: https://doi.org/10.1007/s10457-0180237-3

Diatta EA, Sagna B. 2013. Caractérisation et modes d'exploitation des parcs à Elaeis guineensis, Jacq. en Basse Casamance : cas des villages de Carounate (cassa) et de Djiguinoume (kalounayes). Mémoire de Licence Agroforesterie, Université Assane de Ziguinchor, 49 p.

Diedhiou MAA, Faye E, Ngom D, Touré MA. 2014. Identification et caractérisation floristiques des parcs agroforestiers du terroir insulaire de Mar Fafaco, Sénégal. Journal of Applied Biosciences, 79: 6855-6866.

Gbesso F, Yedomonhan H, Tenté B, Akoegninou A. 2014. Distribution géographique des populations de rôniers (Borassus aethiopum Mart, Arecaceae) et caractérisation phytoécologique de leurs habitats dans la zone soudano-guinéenne du Benin. Journal of Applied Biosciences, 74: 60 $99-6111$.

Girardin O, Sorg JP, Herzog F. 2002. Le vin de palme, aliment et source de revenu pour les populations rurales en Côte d'Ivoire. Schweizerische Zeitschrift fur Forstwesen, 153(4): 123-129. DOI: 10.3188/szf.2002.0123

Gomis ZD. 2014. Les parcs agroforestiers à Elaeis guineensis Jacq. (Palmier à huile): Caractéristiques biophysiques et importance socio-économique à Ouonck (Basse-Casamance). Mémoire de Master 2, Université Assane de Ziguinchor, $79 \mathrm{p}$.

Goudiaby M. 2013. Les parcs agroforestiers en Basse-Casamance : Contribution du Parkia biglobosa (néré) à la réduction des risques de pauvreté des ménages de 
la communauté rurale de Mangagoulack, au Sénégal. Mémoire, Université de Laval, Québec, Canada, $98 \mathrm{p}$.

Graham PH, Vance CP. 2003. Legumes: importance and constraints to greater use. Plant Physiol., 131(3): 872-877.

Manolescu DC. 2009. Niveaux de vitamine A (rétinol et acide rétinoïque) mesurés dans le sang de cordon ombilical et développement rénal des nouveau-nés. Mémoire présenté à la Faculté des Études Supérieures en vue d'obtention du grade de Maîtrise Sciences (M.Sc.) en Nutrition, Université de Montréal, $164 \mathrm{p}$.

Ngom D, Akpo LE, Mbaye T, Charahabil MM, Camara B, Diedhiou AA, Gomis ZD, Sagna B. 2015. Les parcs agroforestiers traditionnels : caractéristiques écologiques et influences sur la riziculture pluviale en zone post-conflit de la Basse Casamance. Projet Banque Mondiale, $22 \mathrm{p}$.

Ngom D. 2014. Perceptions communautaires sur les services écosystémiques d'approvisionnement fournis par le peuplement ligneux de la réserve de Biosphère du Ferlo (Sénégal). Vertigola revue électronique en science de l'environnement, 14 (2) : $17 \mathrm{p}$.
Niang B. 2014. Le palmier à huile en zone urbaine et périurbaine : dynamique générale, aspects socio-économiques culturels dans la ville d'Oussouye, 82 p.

Ofosu-Budu K, Sarpong D. 2013. Croissance de la filière de l'huile de palme au Ghana : implications pour les petits agriculteurs et viabilité à long terme. In Reconstruire le Potentiel Alimentaire de l'Afrique de l'Ouest, Elbehri A (ed). FAO/FIDA : Rome.

Sagna B. 2016. Paramètres structuraux, modes de gestion et importance socioéconomique des parcs agroforestiers à Elaeis guineensis Jacq. (Palmier à huile) en Basse-Casamance. Mémoire de Master 2, Université Assane SECK de Ziguinchor, 55 p.

Sagna B. 2017. Importance socioéconomique de Elaeis guineensis Jacq. (Palmier à huile) en Basse-Casamance (Sénégal). Communication orale, Colloque international sur «Les arbres fruitiers sauvages de l'Afrique de l'ouest», Université Assane Seck Ziguinchor (20 au 22 juillet 2017).

Yombouno A. 2014. Historique de l'exploitation du palmier à huile en Afrique. ADAPE-Guinée, 36 p. 